in vivo $34: 2363-2370(2020)$

doi:10.21873/invivo.12049

\title{
Expansion of Human Dental Pulp Cells In Vitro Under Different Cryopreservation Conditions
}

\author{
MING YAN, OLA A. NADA, LAN KLUWE, MARTIN GOSAU, RALF SMEETS and REINHARD E. FRIEDRICH
}

Department of Oral and Maxillofacial Surgery, University Medical Center Hamburg-Eppendorf, Hamburg, Germany

\begin{abstract}
Background/Aim: To optimize the expansion of human dental pulp cells in vitro by exploring several cryopreservation methodologies. Materials and Methods: The intra-dental pulp tissues from healthy subjects were extracted and divided into three separate tissue segments, which were randomly divided into the three following groups; the fresh group, the 5\% DMSO group, and the 10\% DMSO group. In the fresh group, dental pulp cells were directly cultivated as primary cultures, whereas in the DMSO groups, the dental pulp cells were cultivated from cryopreserved pulp tissue segments one month later. Results: The cell yield and the time it took for the cells to grow out of the pulp tissue and attach to the culture plate varied among the three groups; the 5\% DMSO group was inferior to the fresh group but superior to the $10 \%$ DMSO group $(p<0.05)$. Moreover, no differences were found at the 1st passage amongst the three groups regarding the following aspects ( $p>0.05)$; colony formation rate and cell survival rate. Furthermore, no differences were noted at the $3^{\text {rd }}$ passage regarding the following aspects $(p>0.05)$; proliferation ability, cell growth curve and surface marker expression of dental pulp cells. Conclusion: Five percent DMSO may be the most optimal condition for tissue storage to preserve stem cells in situ.
\end{abstract}

Dental pulp cells are regarded as a convenient source of cells with stem cell characteristics that can be employed for regenerative therapies $(1,2)$. Not only they are a convenient source of cells because of their availabilty from readily available extracted teeth such as wisdom teeth and decidous teeth, but because they are also not associated with any ethical

This article is freely accessible online.

Correspondence to: Prof. Reinhard E. Friedrich, Department of Oral and Maxillofacial Surgery, University Medical Center Hamburg-Eppendorf, Hamburg 20246, Germany. Tel: +49 (0)40741053259, Fax: +49 (0)4074100, e-mail: r. friedrich@uke.de

Key Words: Stem cells, stem cell culture and differentiation, dimethyl sulfoxide, cryopreservation, cell proliferation; osteogenic induction, adipogenic induction. controversies due to either being routinetly prophylactically extracted for the former or naturally exfoliated for the latter (3). Moreover, dental pulp cells are a potent source due to their multi-differentiation potential and high proliferative potential (4-7). Therefore, successful preservation of intra-dental pulp tissue is a prime advantage for future regenerative therapies. Tissue preservation at ultra-low temperatures can be one of the most reliable methods for tissue storage. In the current study, after primary tissue culture, primary cells were stored in cryopreservative medium containing dimethyl sulfoxide DMSO $(8,9)$. Therefore, graded concentrations of DMSO were examined in this study. It has been shown that the addition of an appropriate volume ratio of DMSO to cryopreservative medium can prevent physical damage of cells caused by ice crystals during cryopreservation and thawing. Nevertheless, DMSO does possess cytotoxic effects. Moreover, besides being non-environmentally friendly, it can also directly affect the health of the operator during handling (10-12). Therefore, if the concentration of DMSO used can be reduced, the risk of cytotoxic effects can be reduced to some extent for the sake of more successful cell transplantation therapy applications. This study aimed to explore variations that could possibly occur to stem cell cultivation time, cell yield, as well as cellular activities when stem cells are cultivated from both fresh pulp tissue segments or frozen pulp tissue segments that have been preserved using different cryopreservation conditions. Thereby, coming a step closer to finding the most suitable scheme for storing pulp tissue samples as a convenient and readily available source for cells with stem cell characteristics and hence, lay down the foundation for a reasonable development of a sustainable clinical sample harvesting system that can be used for future cell transplantation applications.

\section{Materials and Methods}

Extraction and cryopreservation of dental pulp tissue: Ten supernumerary and deciduous teeth of healthy children aged 10-15 years were collected from December of 2018 to May of 2019 at the department of Oral and Maxillofacial Surgery of our hospital. Before the surgery, all the patients and/or their respective guardians signed the informed consent, and were instructed to gargle with $1 \%$ hydrogen peroxide solution. The surgery also followed routine intra 
and extra- oral disinfection measures. The teeth were then extracted and placed in culture medium-complete DMEM [(Dulbecco's Modified Eagle Media (cat. no. 11965084, ThermoFisher Scientific, Waltham, MA, USA)] with $10 \%$ fetal bovine serum (FBS, ThermoFisher Scientific, cat. no. 10438034) and penicillin (100 $\mathrm{U} / \mathrm{ml}) /$ streptomycin $(100 \mu \mathrm{g} / \mathrm{ml})$ (cat. no. 15140-148, Gibco BRL, Carlsbad, CA, USA) $4^{\circ} \mathrm{C}$. Experiments were conducted within $4 \mathrm{~h}$ thereafter. Teeth were rinsed with $4^{\circ} \mathrm{C}$ PBS (Gibco, cat. 18912-014) solution (including $2 \times 10^{5} \mathrm{U} / 1$ penicillin and streptomycin) from the crown to the root repeatedly for a duration of 5-10 min. Teeth were then cut open to expose the pulp using a hammer. Thereafter, the intra-dental pulp tissues were immersed in culture medium and were then cut into $0.5 \mathrm{~mm}^{3}$ tissue pieces.

The pulp tissue pieces were randomly divided into a fresh group and two frozen groups. For the frozen groups, 5\% and 10\% DMSO (cat. no. D8418, Sigma-Aldrich, St. Louis, MO, USA) medium were used to suspend the pulp tissue pieces to be stored in the $-196^{\circ} \mathrm{C}$ liquid nitrogen for 1 month after having been initially stored in the refrigerator at $4^{\circ} \mathrm{C}$ for $1 \mathrm{~h}$. Whereas, for the fresh group, the dental pulp cells were immediately cultivated.

Culture of human dental pulp cells: At the time of cultivation, tissues from each group were evenly distributed and spread in three $3.5 \mathrm{~cm}$ diameter culture dishes. Each tissue piece was dropped with $100 \mu \mathrm{l}$ DMEM medium to ensure its attachment to the culture surface. The culture dishes were incubated at $37^{\circ} \mathrm{C}$ in a $5 \% \mathrm{CO}_{2}$ humidified incubator. After 2 days, cell attachment and growth were observed under an inverted microscope, followed by the gradual formation of colonies until the desired level of confluency was reached. Then, the cells were harvested from the culture surface using $0.05 \%$ TrypsinEDTA (ThermoFisher; cat. no. 25300-054) and were counted using cell-count boards. The cells were passaged at a density of $5 \times 10^{3} / \mathrm{cm}^{2}$.

Observation of cell morphology: Primary cells were observed under an inverted microscope, and the time elapsed from the placement of tissue pieces to cellular outgrowth and attachment was noted for each of the three groups.

Calculation of cell yield/harvest: Upon reaching a state of confluency, primary cells of each group were subpassaged with $0.05 \%$ trypsin at $37^{\circ} \mathrm{C}$ for $1-2 \mathrm{~min}$. The process of trypsinization was stopped when cellular attachment was seen to be clearly disrupted by the appearance of floating cells in the medium. After centrifugation at $400 \times \mathrm{g}$ for $8 \mathrm{~min}$ at room temperature, the primary dental pulp cells were resuspended in complete DMEM culture medium and were counted as a cell suspension under the microscope using cell counting plates/hemocytometer. The primary cell yield was calculated using the following equation.

Primary cell yield=Total cell counted/4 $\times$ dilution factor $\times$ $10^{4} \times$ volume of cell suspension (13).

Colony forming efficiency: The first generation of cells $\left(2 \times 10^{3}\right.$ cells $)$ of each group was cultured into a $10 \mathrm{~cm}$ diameter culture dish for 14 days. After which the culture medium was discarded and the newly formed dental pulp colonies were fixed with $95 \%$ methanol for $15 \mathrm{~min}$, followed by staining with $3 \%$ crystal violet dyes for 2 min, which was then rapidly decolorized using methanol, followed by PBS washes to remove residual crystal violet dye solution. Single colonies with a cell count greater than 50 , were recorded as successfully formed colonies. Three parallel samples were set up in each group and counted twice. The Colony forming efficiency $\%$ was calculated using the following equation:

Colony forming efficiency $(\%)=$ number of successfully formed colonies/number of seeded cells $\times 100$ (14).

Cell survival rate: The $1^{\text {st }}$ generation of cells from each group was observed under an inverted microscope after being stained with $0.4 \%$ Trypan Blue (Sigma-Aldrich). Dead cells were stained blue whereas surviving cells were left unstained. The survival cell rate of 500 cells was rapidly counted within $5 \mathrm{~min}$ and the cell survival rate was calculated using the following equation:

Cell survival rate $(\%)=$ number of unstained surviving cell/ $500 \times 100(15)$

Evaluation of cell proliferation using the MTS assay: Dental pulp cells which were passaged for 3 generations were made into cell suspensions per generation and their proliferation was measured using the MTS (3-(4, 5-dimethylthiazol-2-yl)-5-(3-carboxymethoxyphenyl)2-(4-sulfophenyl)-2H-tetrazolium, inner salt) assay (cat. no. G3582, Promega, Madison, WI, USA). Cell were seeded at concentrations of $5 \times 10^{9} / 1$ into 96 -well plates for 8 days and incubated at $37^{\circ} \mathrm{C}$ and $5 \%$ $\mathrm{CO}_{2}$. Cells from 5 wells were subjected to MTS colorimetric analysis in each group every day for 8 consecutive days. The proliferative readings were determined using UV spectrophotometry at a wavelength of $490 \mathrm{~nm}$ using a nanodrop spectrophotometer (Thermo Fisher Scientific, Waltham, MA, USA).

Determination of the immunophenotype of cells using specific surface markers: After the $3^{\text {rd }}$ generation cells were filtered using a $70 \mu \mathrm{m}$ filter, the cells were re-suspended in cold PBS at a concentration of $1 \times 10^{9} / 1$. Surface markers of $3^{\text {rd }}$ generation cells were identified by flow cytometry using antibodies against STRO-1 (cat. no. 14-6688-82; Thermo Fisher Scientific, Inc.), CD105 (cat. no. ab156756; Abcam, Cambridge, UK), CD90 (cat. no. ab139364; Abcam), CD34 (cat. no. 560941; BD Biosciences, San Jose, CA, USA), and CD45 (cat. no. MCA2035S; Bio-Rad Laboratories, Inc, Hercules, CA, USA) and antibodies (all 1:500) was employed to detect the phenotype of cells.

\section{Multidifferentiation potential}

Adipogenic differentiation: Third generation dental pulp cells were seeded at a density of $8 \times 10^{3} / \mathrm{cm}^{2}$ onto $3.5 \mathrm{~cm}$ culture dishes. As cells reached $70 \%-80 \%$ confluency, growth culture medium was replaced by adipogenic inductive medium for a duration of 21 days. Medium was changed regularly 2-3 times per week. As the induction period advanced, lipid droplets were observed in the cytoplasm of cells. Following the 21 day period, inductive medium was discarded and cells were washed twice with PBS and then fixed by $4 \%$ paraformaldehyde (PFA; Electron Microscopy Sciences, Fort Washington, PA, USA) for 15 min. Following fixation, cells were washed twice with PBS and then stained with $0.5 \%$ oil red $\mathrm{O}$ working solution (Sigma, cat. no. 00625, 0.4\%) at room temperature for 5-10 min. Stained lipid droplets in the cytoplasm were then observed under the microscope and photographed.

Quantitative analysis of adipogenic differentiation: Cultures stained with oil red O stain were carefully washed 3 times with distilled water. To dissolve the lipid droplets, $500 \mu \mathrm{l}$ isopropanol (Sigma- 
Table I. Primer sequences and reaction conditions to measure adipogenic and osteogenic induced gene expression.

\begin{tabular}{|c|c|c|c|}
\hline Primer & Sequence of upstream and downstream & $\mathrm{Ta}\left({ }^{\circ} \mathrm{C}\right)$ & Length product (bp) \\
\hline \multicolumn{4}{|l|}{ Adipogenic induced gene } \\
\hline \multirow[t]{2}{*}{ Lipoprotein lipase } & Upstream: 5'-GAC TGA GAG TGA AAC CCA TAC-3' & & \\
\hline & Downstream: 5'-CCC TAG AAC AGA AG ATC ACC-3' & 54 & 290 \\
\hline Peroxisome Proliferator-Activated & Upstream: 5'-GCT TCT GGA TTT CAC TAT GG-3' & & \\
\hline Receptor Coactivator $\gamma$ & Downstream: 5'-AAA CCT GAT GGC ATT ATG AG-3' & 54 & 195 \\
\hline \multicolumn{4}{|l|}{ Osteogenic induced gene } \\
\hline \multirow[t]{2}{*}{ Type I collagen } & Upstream: 5'-GGA CAC AAT GGA TTG CAA GG-3' & & \\
\hline & Downstream: 5'-TAA CCA CTG CTC CAC TCT GG-3' & 58 & 461 \\
\hline \multirow[t]{2}{*}{ Osteocalcin } & Upstream: 5'-ATG AGA GCC CTC ACA CTC CTC-3' & & \\
\hline & Downstream: 5'-GCC GTA GAA GCG CCG ATA GGC-3' & 57 & 293 \\
\hline \multicolumn{4}{|l|}{ Internal reference } \\
\hline \multirow[t]{2}{*}{ GAPDH } & Upstream: 5'-ATC ACC ATC TCC AGG AGC G-3' & & \\
\hline & Downstream: 5'-GTT CTT CCA CCA CTT CGT CC -3 & 55 & 573 \\
\hline
\end{tabular}

Aldrich, cat. no. 278475) was added into each well. The dissolved stain solution was then mixed and evenly distributed in 96-well plate. Finally, the absorbance value was read at a wavelength of 540 $\mathrm{nm}$ on the spectrophotometer.

Osteogenic differentiation: Third generation dental pulp cells at a density of $8 \times 10^{3} / \mathrm{cm}^{2}$ were seeded onto $3.5 \mathrm{~cm}$ culture dishes. As cells reached $70 \%-80 \%$ confluency, growth culture medium was replaced by osteogenic inductive medium for a duration of 21 days. Medium was changed regularly 2-3 times per week. As the induction period advanced, calcified nodules were observed in the cytoplasm of cells. Following the 21 day period, inductive medium was discarded and cells were washed twice using DPBS and then fixed by $90 \%$ ethanol (EtOH; Sigma-Aldrich, cat. no. 270741) for 1 h. Following fixation, cells were washed twice with PBS and then stained with $2 \%$ Alizarin red S (cat. no. 130-22-3; Sigma-Aldrich) stain at room temperature for 5-10 min. Stained calcified nodules in the cytoplasm were then observed under the microscope and photographed.

Quantitative analysis of osteogenic differentiation: Cultures stained with Alizarin red $\mathrm{S}$ were carefully washed 3 times with distilled water. To dissolve the calcified nodules, $500 \mu \mathrm{l}$ of $10 \%$ acetic acid (Sigma-Aldrich, cat. no. 33209) were added into each well. Equal volume of $10 \%$ ammonium hydroxide was then added to neutralize the acetic acid. The resultant solution was mixed and evenly distributed in the 96-well plate. Finally, the absorbance value was read at a wavelength of $405 \mathrm{~nm}$ using a spectrophotometer.

In the aforementioned differentiation experiments, 3 parallel samples were set up for each group, and 5 well replicates were analyzed for each sample. The whole experiment was repeated twice.

Reverse transcription PCR to measure adipogenic and osteogenic induced gene expression: To extract total RNA from cells, $1 \mathrm{ml}$ Trizol reagent (Invitrogen, cat no. 15596-018) was added to every $1 \times 10^{6}$ second-generation cells. The expression of the adipogenic specific genes Lipoprotein Lipase and Peroxisome proliferatoractivated receptor- (PPAR- $\gamma$ ) and the osteogenic specific genes type I collagen and osteocalcin was measured by reverse transcription PCR. GAPDH was used as a reference gene. Primer sequences and reaction products are depicted in Table I.
The conditions of the PCR reaction were: $94^{\circ} \mathrm{C}$ for $4 \mathrm{~min} ; 94^{\circ} \mathrm{C}$ for $30 \mathrm{~s}, 54-58^{\circ} \mathrm{C}$ for $30 \mathrm{~s}, 72^{\circ} \mathrm{C}$ for $1 \mathrm{~min} ; 35-40$ cycles; $72^{\circ} \mathrm{C}$ for 7 min, $4^{\circ} \mathrm{C}$. Amplified PCR products were then evaluated by $2 \%$ agarose gel electrophoresis and $2 \%$ ethidium bromide staining. Finally, gel imaging was obtained under UV light. The relative expression of adipogenic and osteogenic gene mRNAs was measured by reverse transcription PCR. (Adipogenic specific genes: Lipoprotein Lipase and Peroxisome proliferator-activated receptor$($ PPAR- $\gamma)$; Osteogenic specific genes: type I collagen and osteocalcin).

Ethics approval and consent to participate: Administrative permissions were acquired by our team to access the data used in our research. The study protocol was approved by the Hamburg University ethics committee that approved the study. No: REC $1712 / 5 / 2008$. Accordingly, all teeth were coded with number and all personal identification of the patients were removed. All parent or guardian of participants provided written informed consent for using their teeth, which otherwise would have been discarded as waste.

Statistical analysis: Student $t$-test was used to analyze differences in mean values between the three groups. $p$-Values less than 0.05 were considered statistically significant. SPSS 17.0 software (SPSS Inc., Chicago, IL, USA) was used for statistical analysis.

\section{Results}

Effects of different cryopreservation conditions on primary cell cultivation and expansion. Morphologically cultured dental pulp cells, observed under the inverted microscope, appeared as either spindle-shaped, triangular or long spindleshaped, with an abundant cytoplasm and a clear nucleus. No clear difference was noted between the two groups, as shown in Figure 1A. However, the time elapsed for cellular outgrowth and attachment was significantly shorter in the fresh group when compared to the frozen group $(p<0.05)$, as shown in Figure 1B. 
A
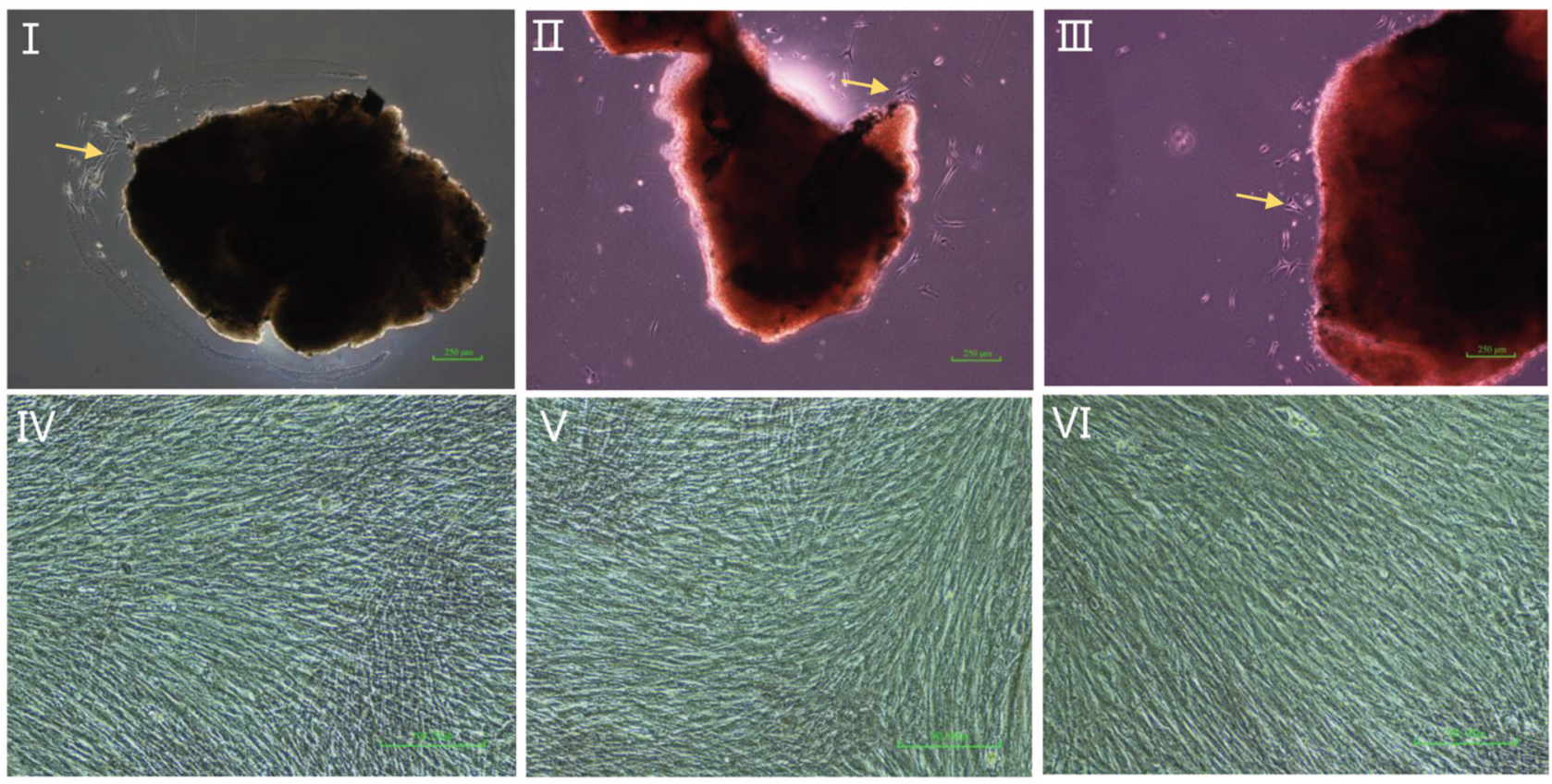

B

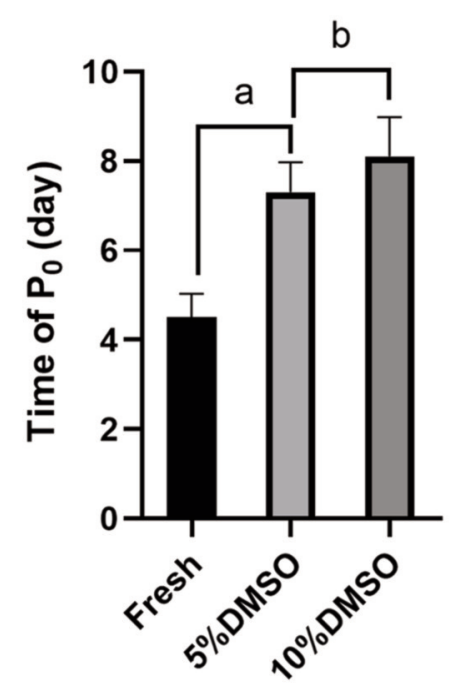

Figure 1. Morphology of pulp cells under different cryopreservation conditions. A: Morphology of primary and $3^{\text {rd }}$ generation/passage dental pulp cells. I-III: Primary cells from different groups grew out of the tissue mass (arrow, x40); IV-VI: Dental pulp cells morphology at the 3 rd passage/generation - depicting both fresh and frozen groups. B: Time of appearance of primary cells: Time of appearance of primary cells in the fresh group was significantly lower than that in frozen groups $\left({ }^{a} p<0.05\right)$; Time of appearance of primary cells in the $5 \%$ DMSO group was significantly lower than that in the 10\% DMSO group $\left({ }^{a b} p<0.05\right)$. DMSO cryopreservation of primary pulp cells resulted in increased time to grow out of the tissue.

Cryopreservation effects on the primary dental pulp cell yield/cell harvest. The number of primary dental pulp cells in both frozen groups was significantly lower than that in the fresh group $\left({ }^{\mathrm{a}} p<0.05\right)$. Furthermore, the lowest dental pulp cell yield number was observed in the 10\% DMSO group only $(3.625 \pm 0.375) \times 10^{5}\left({ }^{b} p<0.05\right)$ (Figure 2$)$.
Cryopreservation effects on stem cell characteristics of primary dental pulp cells. Flow cytometric analysis was used to examine the expression of stem cell markers in the three groups. All groups showed greater than $70 \%$ positive expression of $\mathrm{CD} 105$ and $\mathrm{CD} 90$, and $8 \%-16 \%$ positive expression of STRO-1. The expression of CD34 and CD45 


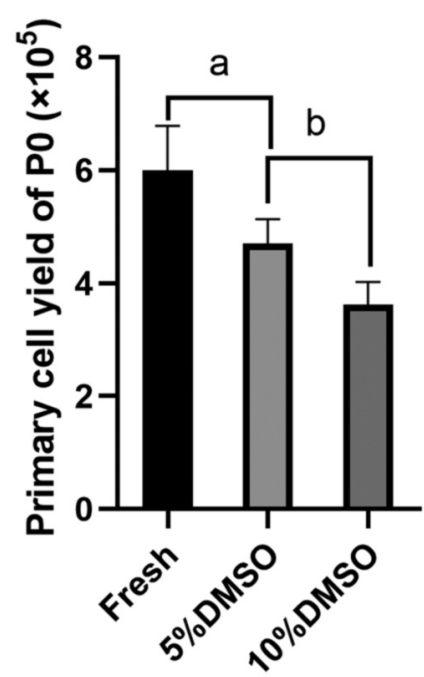

Figure 2. Cryopreservation effects on primary dental pulp cell yield. A Dose-dependent inverse association between DMSO and dental pulp cell yield is observed.

was less than $5 \%$ in all groups. Nevertheless, there was no significant difference in the positive expression rates of CD105, CD90, CD34, CD45 and STRO-1 between the three groups $(p<0.05)$ (Figure 3$)$.

Cryopreservation effects on primary dental pulp cell proliferation and renewal. Compared to the fresh group, no significant differences were noted regarding the colony forming efficiency, the survival rate of first generation dental pulp cells, and the growth curve third generation dental pulp cells $(p>0.05)$ (Figure 4$)$.

Cryopreservation effects on the multidifferentiation potential of dental pulp cells. Cellular products in the form of oil droplets and calcific deposits resulting from adipogenic and osteogenic induction, respectively, were subjected to staining protocols followed by spectrophotometric quantification. Moreover, expression of adipogenic and osteogenic specific genes was also measured. The results showed that regardless of the group, dental pulp cells possess an inherent ability for both adipogenic and osteogenic differentiation $(p>0.05)$ (Figure 5).

\section{Discussion}

Stem cells are undifferentiated cells with a potent selfrenewal ability and a multidifferentiation potential. Stem cells exist not only in embryos but also in adult tissues as well. Accordingly, they can be categorized into embryonic stem cells and adult stem cells. Dental pulp stem cells (DPSCs) are a type of adult stem cells derived from the

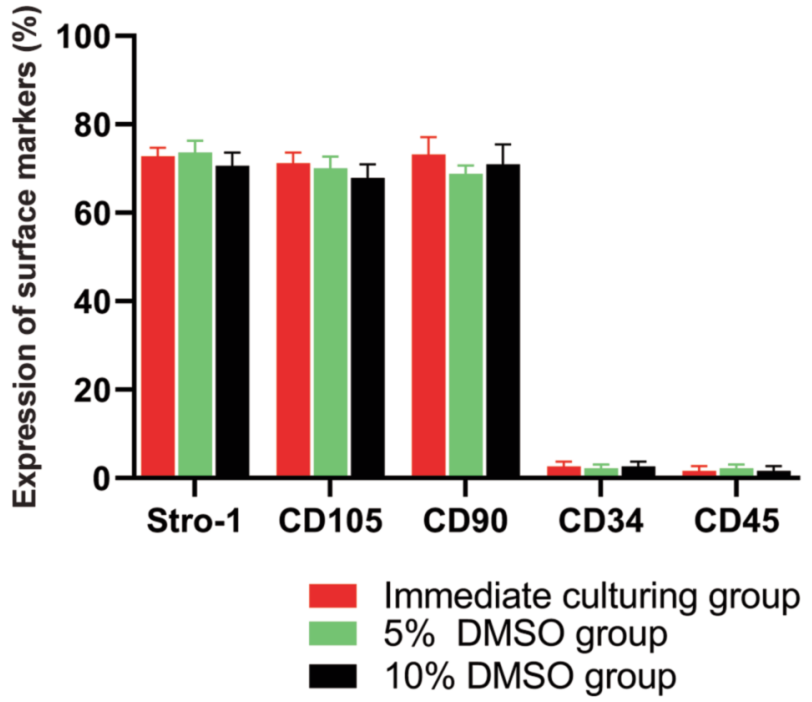

Figure 3. Expression of dental pulp cell surface markers in each group.

dental pulp tissue. They, therefore possess a self-renewal ability and a multi-differentiation potential. Depending on the regulation of their microenvironment, they can differentiate into osteoblasts, odontoblasts, adipocytes, muscle cells and nerve cells. DPSCs play an important role in tooth regeneration, nerve repair, and bone tissue engineering. Therefore, dental pulp is regarded as a convenient and widely available source of stem cells for tissue regeneration and thereby, translational medicine.

Due to the convenience of their isolation from exfoliated primary or routinely extracted permanent teeth, they can be produced in sufficient quantities for various applications (16). Therefore, a problem to be solved at the present time is to establish an efficient and reliable banking system for this readily available and valuable tissue. Studies have shown that cryopreservation is an effective method for longterm preservation of biological tissue samples (17). However, in order to extract biologically active stem cells from frozen tissues, tissue samples cannot be simply placed in liquid nitrogen. A tissue cryopreservation should be developed and adjusted accordingly to ensure successful future stem cell cultivation and application (18).

DMSO is the main chemical reagent used for cell cryopreservation due to its protective effects against osmotic cell rupture. At severely low temperatures, as low as $-200^{\circ} \mathrm{C}$, DMSO can prevent damage caused by ice crystal formation and osmotic pressure changes. However, although DMSO is currently the best cell cryoprotectant, it is also a toxic chemical reagent, mainly manifested during the process of cell recovery and culture (19). Studies have shown that when 

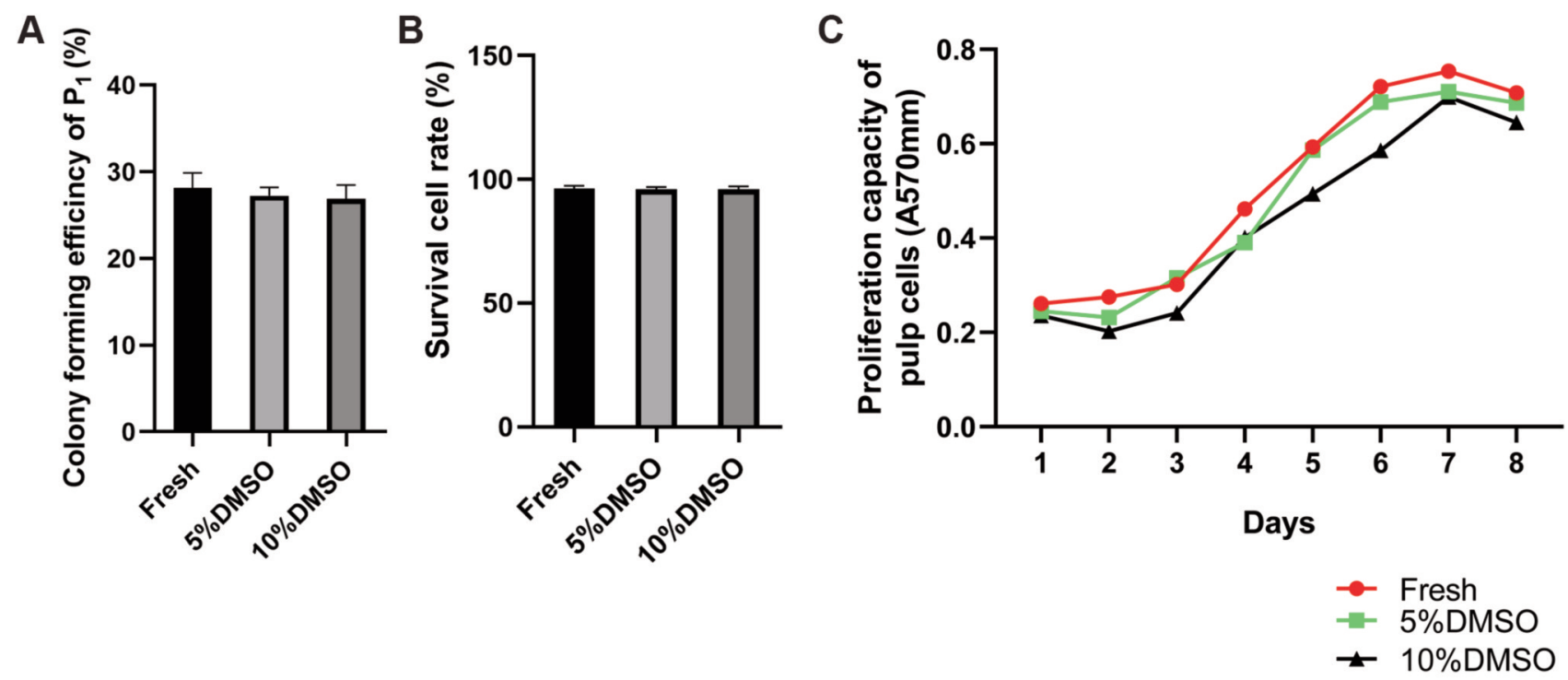

Figure 4. Self-renewal and proliferation capacity of dental pulp cells in each group. A: Colony forming efficiency of primary dental pulp cells in each group; B: Survival rate of primary dental pulp cells in each group; C: Cell growth curve of primary dental pulp cells in each group (MTS).

the concentration of DMSO in the culture solution is $10 \%$, cell growth inhibition rate is nearly $100 \%$. Whereas when its concentration is $0.1 \%$, the inhibition rate drops to $35 \%$. Furthermore, even when the concentration of DMSO is $0.004 \%$, it continues to inhibit cellular growth (20). Moreover, as a "universal solvent" DMSO has also potent skin permeation and volatilization characteristics, making it a hazard for the researcher. Therefore, if the dosage and frequencies of DMSO usage in cell freezing and thawing procedures can be reduced, the operation safety factor can be also improved, in addition to reducing risks associated with stem cell transplantation therapies.

Therefore, there is a shift to switch from cryopreservation at a cellular level to cryopreservation at the tissue level. While doing so, the traditional $10 \%$ DMSO addition ratio will also be reduced to $5 \%$, in an attempt to optimize cryopreservation and obtain the lowest risk: benefit ratio possible.

Our results showed that the time elapsed for primary cells to grow out of tissue pieces was significantly longer in both cryopreserved groups when compared to the positive control group. However, when comparing data among the 2 cryopreserved groups, the 5\% DMSO group displayed significantly shorter time lapse for cellular outgrowth in comparison to the $10 \%$ DMSO group $(p<0.05)$.

The aforementioned results are based on primary cells and do show the influence of cryopreservation and thawing on the initial stage of in vitro cell cultivation, which cause a prolongation in the time needed for cellular outgrowth and cultivation, and also a reduction in the total cell harvest or yield.
However, a series of experiments in the later period adopt the third generation of cells as the research object, because the cells can be naturally purified after passage, which is the suitable cell generation for clinical treatment recognized by the research. Therefore, the experimental cells can not only ensure the number of experimental cells, but also are very representative once it is proved that their biological activity will not be changed due to the freezing of donor tissue samples, which can lay a theoretical foundation for future clinical transplantation treatment and improve the safety of treatment.

Our findings confirmed the lack of significant differences in basic properties and stem cells characteristics amongst the cryopreservation conditions and the freshly extracted dental pulp cells $(p>0.05)$. Our results are consistent with those of previous research (21).

\section{Conclusion}

In conclusion, cryopreservation can have a hazardous effect on the extraction and harvest of primary dental pulp cells, but an appropriate reduction in the ratio of DMSO can attenuate this effect. However, cryopreservation does not affect the biological activity of the cultured dental pulp stem cells and can therefore be safely applied to cellular transplantation therapies. In conclusion, judging from the total cell yield and the time required for successful in vitro cultures, the 5\% DMSO was found to be superior to $10 \%$ DMSO and can thereby be regarded as an optimal option for dental pulp tissue storage. Moreover, considering the total 
A
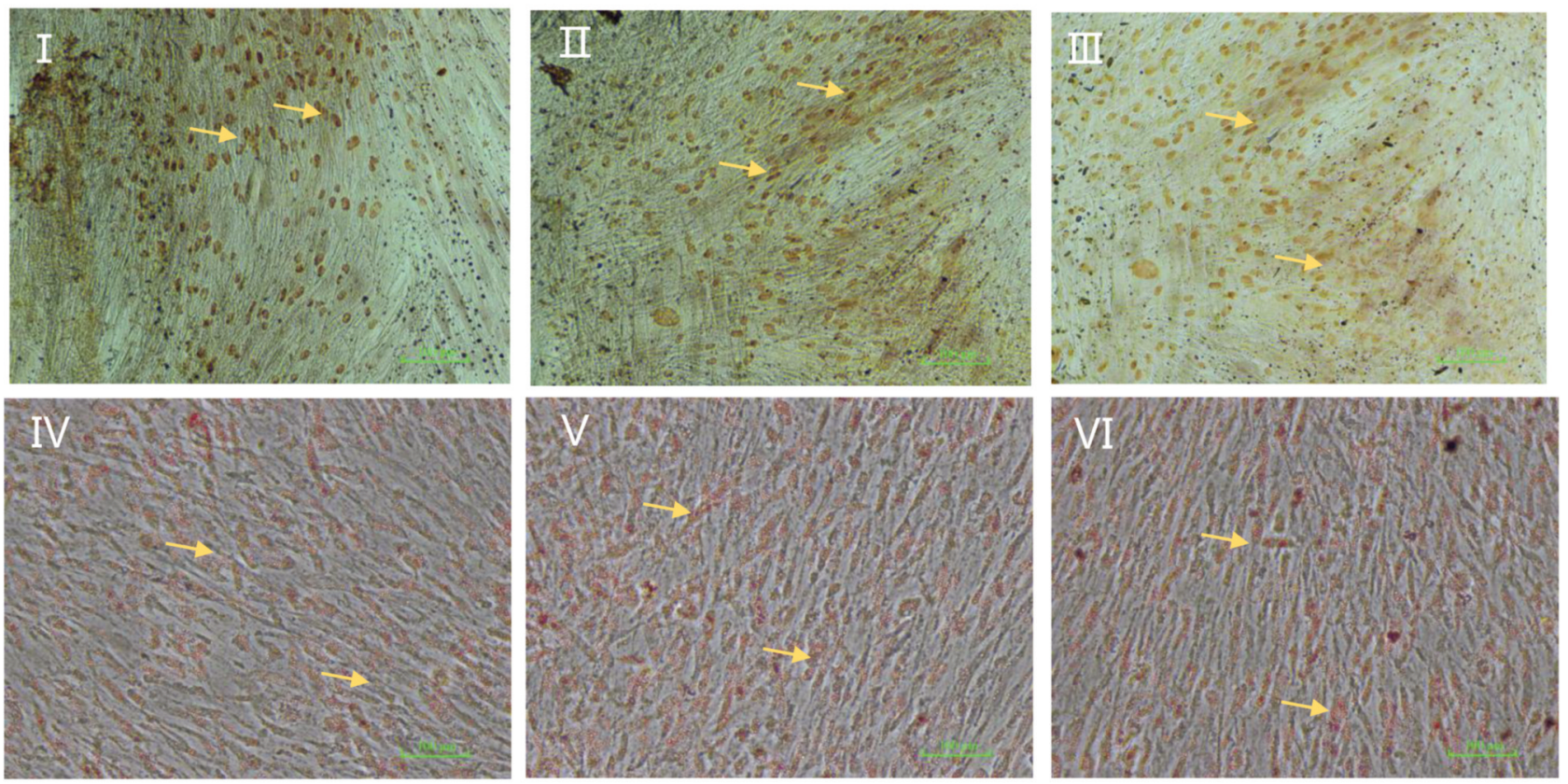

B

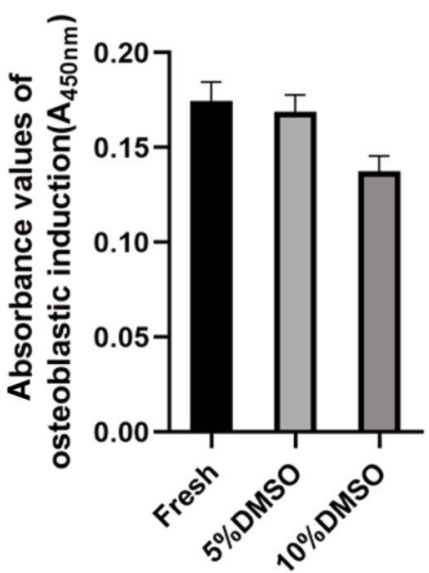

C

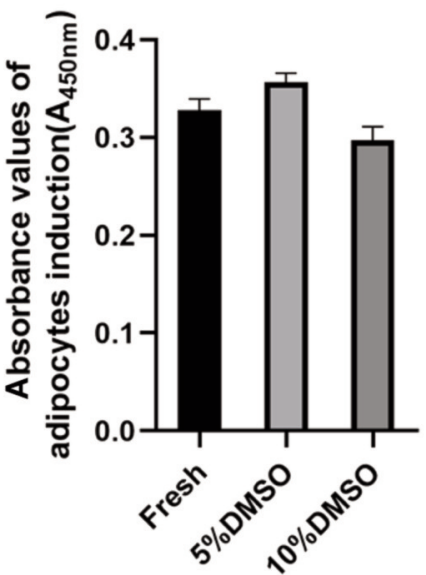

D

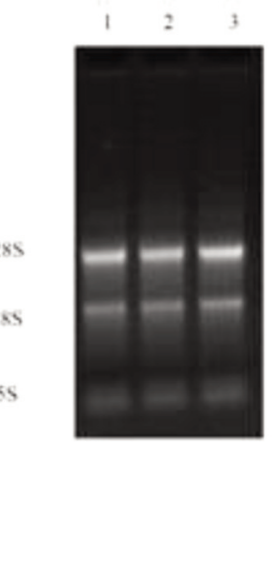

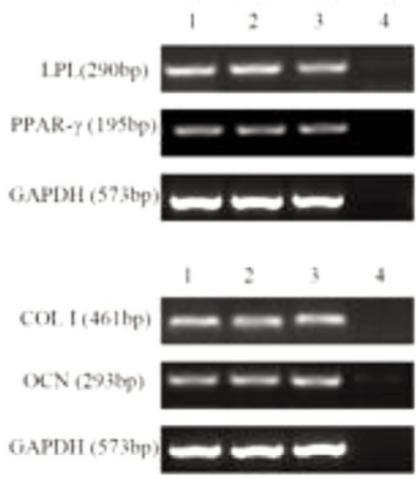

Figure 5. Multi-differentiation potential of dental pulp cells from each group. A: Adipogenic and osteogenic differentiation potential of $3^{\text {rd }}$ generation dental pulp cells in each group - Oil Red $O$ and Alizarin Red S staining results, respectively. I-III: Osteogenic differentiation (2\% Alizarin red stain, $\times 100)$. I: Fresh group; II: 5\% DMSO group; III: 10\% DMSO group. IV-VI: Adipogenic differentiation (0.5\% Oil red o stain, x100): IV: Fresh group; V: $5 \%$ DMSO group; VI: 10\% DMSO group. B: The absorbance values (A values) after osteogenic differentiation by Alizarin Red S (ARS) staining of 3 rd generation dental pulp cells in each group. C: The absorbance values (A values) after adipogenic differentiation by Oil Red $O$ staining of $3^{\text {rd }}$ generation dental pulp cells in each group. I: Electrophoresis of RNA: 1:Fresh group; 2:5\% DMSO group; 3:10\% DMSO group. II: Electrophoresis of adipogenic specific gene expression in dental pulp cells from each group:1:Fresh group; 2:5\% DMSO group; 3:10\% DMSO group; 4:Negative control. III: Electrophoresis of osteogenic specific gene expression of dental pulp cells in each group:1:Fresh group; 2:5\% DMSO group; 3:10\% DMSO group; 4:Negative control. D: The expression of specific adipogenic and osteogenic genes as a result of the respective differentiation by RT-PCR. Note: Dental pulp cells cultivated and expanded from the three groups demonstrated stem (progenitor) cell characteristics of proliferation, self-renewal and multipotentiality.

volume of DMSO, it is suggested to opt for tissue cryopreservation as a whole rather than cellular cryopreservation in efforts to reduce the direct toxicity effects of DMSO to cells, thereby decreasing possible cell losses in order to maximize cell yield for cell transplantation therapies. 


\section{Conflicts of Interest}

The Authors declare that they have no competing interests in relation to this study.

\section{Authors' Contributions}

MY: conceived the study, supervised the experiments and drafted the manuscript. LK: performed data collection and drafted the manuscript. REF: designed the experiments and drafted the manuscript. ON: data evaluation, medical writing and editorial assistance in preparing this manuscript for publication. RS: performed the data collection. MG: analyzed the data and revised the manuscript. All Authors have read and approved the manuscript.

\section{References}

1 Gronthos S, Mankani M, Brahim J, Robey PG and Shi S: Postnatal human dental pulp stem cells (dpses) in vitro and in vivo. Proc Natl Acad Sci U S A 97(25): 13625-13630, 2000. PMID: 11087820. DOI: 10.1073/pnas.240309797

2 Kabir R, Gupta M, Aggarwal A, Sharma D, Sarin A and Kola MZ: Imperative role of dental pulp stem cells in regenerative therapies: A systematic review. Niger J Surg 20(1): 1-8, 2014. PMID: 24665194. DOI: 10.4103/1117-6806.127092

3 Miura M, Gronthos S, Zhao M, Lu B, Fisher LW, Robey PG and Shi S: Shed: Stem cells from human exfoliated deciduous teeth. Proc Natl Acad Sci USA 100(10): 5807-5812, 2003. PMID: 12716973. DOI: 10.1073/pnas.0937635100

4 Laino G, d'Aquino R, Graziano A, Lanza V, Carinci F, Naro F, Pirozzi $G$ and Papaccio G: A new population of human adult dental pulp stem cells: A useful source of living autologous fibrous bone tissue (lab). J Bone Miner Res 20(8): 1394-1402, 2005. PMID: 16007337. DOI: 10.1359/JBMR.050325

5 d'Aquino R, Graziano A, Sampaolesi M, Laino G, Pirozzi G, De Rosa A and Papaccio G: Human postnatal dental pulp cells codifferentiate into osteoblasts and endotheliocytes: A pivotal synergy leading to adult bone tissue formation. Cell Death Differ 14(6): 1162-1171, 2007. PMID: 17347663. DOI: 10.1038/sj.cdd.4402121

6 Arthur A, Rychkov G, Shi S, Koblar SA and Gronthos S: Adult human dental pulp stem cells differentiate toward functionally active neurons under appropriate environmental cues. Stem Cells 26(7): 1787-1795, 2008. PMID: 18499892. DOI: 10.1634/ stemcells.2007-0979

7 Stevens A, Zuliani T, Olejnik C, LeRoy H, Obriot H, Kerr-Conte J, Formstecher P, Bailliez Y and Polakowska RR: Human dental pulp stem cells differentiate into neural crest-derived melanocytes and have label-retaining and sphere-forming abilities. Stem Cells Dev 17(6): 1175-1184, 2008. PMID: 18393638. DOI: $10.1089 / \mathrm{scd} .2008 .0012$

8 Fabricant L, Kiraly L, Wiles C, Differding J, Underwood S, Deloughery $\mathrm{T}$ and Schreiber M: Cryopreserved deglycerolized blood is safe and achieves superior tissue oxygenation compared with refrigerated red blood cells: A prospective randomized pilot study. J Trauma Acute Care Surg 74(2): 371-376; discussion 376377, 2013. PMID: 23354227. DOI: 10.1097/TA.0b013e31827e1d40

9 Wanderley LS, Luz HK, Faustino LR, Lima IM, Lopes CA, Silva AR, Bao SN, Campello CC, Rodrigues AP and de Figueiredo JR: Ultrastructural features of agouti (dasyprocta aguti) preantral follicles cryopreserved using dimethyl sulfoxide, ethylene glycol and propanediol. Theriogenology 77(2): 260-267, 2012. PMID: 21924476. DOI: 10.1016/j.theriogenology.2011.07.038

10 Melkonyan H, Sorg C and Klempt M: Electroporation efficiency in mammalian cells is increased by dimethyl sulfoxide (dmso). Nucleic Acids Res 24(21): 4356-4357, 1996. PMID: 8932394. DOI: $10.1093 / \mathrm{nar} / 24.21 .4356$

11 Koike M, Ishino K, Kohno Y, Tachikawa T, Kartasova T, Kuroki T and Huh N: Dmso induces apoptosis in sv40-transformed human keratinocytes, but not in normal keratinocytes. Cancer Lett 108(2): 185-193, 1996. PMID: 8973593. DOI: 10.1016/s03043835(96)04408-4

12 Yang C, Madhu V, Thomas C, Yang X, Du X, Dighe AS and Cui Q: Inhibition of differentiation and function of osteoclasts by dimethyl sulfoxide (dmso). Cell Tissue Res 362(3): 577-585, 2015. PMID: 26224539. DOI: 10.1007/s00441-015-2245-1

13 Abusin GA, Abu-Arja RF, Gingrich RD, Silverman MD, Zamba GK and Schlueter AJ: An algorithm for utilizing peripheral blood cd34 count as a predictor of the need for plerixafor in autologous stem cell mobilization-cost-effectiveness analysis. J Clin Apher 28(4): 293-300, 2013. PMID: 23426644. DOI: 10.1002/jca.21256

14 Hatlapatka T, Moretti P, Lavrentieva A, Hass R, Marquardt N, Jacobs R and Kasper C: Optimization of culture conditions for the expansion of umbilical cord-derived mesenchymal stem or stromal cell-like cells using xeno-free culture conditions. Tissue Eng Part C Methods 17(4): 485-493, 2011. PMID: 21166520. DOI: 10.1089/ten.TEC.2010.0406

15 Im DJ, Noh J, Yi NW, Park J and Kang IS: Influences of electric field on living cells in a charged water-in-oil droplet under electrophoretic actuation. Biomicrofluidics 5(4): 44112-4411210, 2011. PMID: 22662063. DOI: 10.1063/1.3665222

16 Aydin S and Sahin F: Stem cells derived from dental tissues. Adv Exp Med Biol 1144: 123-132, 2019. PMID: 30635857. DOI: 10.1007/5584_2018_333

17 Mirabet V, Alvarez M, Solves P, Ocete D and Gimeno C: Use of liquid nitrogen during storage in a cell and tissue bank: Contamination risk and effect on the detectability of potential viral contaminants. Cryobiology 64(2): 121-123, 2012. PMID: 22222678. DOI: 10.1016/j.cryobiol.2011.12.005

18 Seo BM, Miura M, Sonoyama W, Coppe C, Stanyon R and Shi S: Recovery of stem cells from cryopreserved periodontal ligament. J Dent Res 84(10): 907-912, 2005. PMID: 16183789. DOI: $10.1177 / 154405910508401007$

19 Nishigaki T, Teramura Y, Nasu A, Takada K, Toguchida J and Iwata $\mathrm{H}$ : Highly efficient cryopreservation of human induced pluripotent stem cells using a dimethyl sulfoxide-free solution. Int J Dev Biol 55(3): 305-311, 2011. PMID: 21710436. DOI: 10.1387/ijdb.103145tn

20 Han DL, Liu KQ, Guo SS, Zhu HL, Huang C and Wang BH: [Dose-effect relationship of dmso and tween 80 influencing the growth and viability of murine bone marrow-derived cells in vitro]. Zhongguo Shi Yan Xue Ye Xue Za Zhi 16(2): 377-380, 2008. PMID: 18426669.

21 Balci D and Can A: The assessment of cryopreservation conditions for human umbilical cord stroma-derived mesenchymal stem cells towards a potential use for stem cell banking. Curr Stem Cell Res Ther 8(1): 60-72, 2013. PMID: 23270628. DOI: $10.2174 / 1574888 \times 11308010008$

Received June 12, 2020

Revised July 3, 2020

Accepted July 6, 2020 